\title{
Underwater Discharge Phenomena in Inhomogeneous Electric Fields Caused by Impulse Voltages
}

\author{
Bok-Hee Lee ${ }^{\dagger}$, Dong-Seong Kim* and Jong-Hyuk Choi*
}

\begin{abstract}
The paper describes the electrical and optical properties of underwater discharges in highly inhomogeneous electric fields caused by $1.2 / 50 \mu$ s impulse voltages as functions of the polarity and amplitude of the applied voltage, and various water conductivities. The electric fields are formed by a point-to-plane electrode system. The formation of air bubbles is associated with a thermal process of the water located at the tip of the needle electrode, and streamer coronas can be initiated in the air bubbles and propagated through the test gap with stepped leaders. The fastest streamer channel experiences the final jump across the test gap. The negative streamer channels not only have more branches but are also more widely spread out than the positive streamer channels. The propagation velocity of the positive streamer is much faster than that of the negative one and, in fact, both these velocities are independent of the water conductivity; in addition the time-lag to breakdown is insensitive to water conductivity. The higher the water conductivity the larger the pre-breakdown energy, therefore, the ionic currents do not contribute to the initiation and propagation of the underwater discharges in the test conditions considered.
\end{abstract}

Keywords: Underwater discharge, Impulse voltage, Streamer corona, Stepped leader, Time-lag to breakdown, Pre-breakdown, Breakdown voltage-time curve

\section{Introduction}

Recent research into pulsed power technology has led to considerable interest in conduction and breakdown phenomena in water, particularly in its use as a working media for installations of electrical discharge technologies. Pulsed conduction and electrical discharges in water are widely used in various industrial and environmental applications, such as energy storage, switching in pulsed power systems, water purification and sterilization [1]-[5]. The electrical breakdown phenomena within liquids have been investigated in detail, particularly in the field of electrical insulation. However, investigations into the mechanisms and the nature of pulsed streamer discharges in liquids have not been so numerous. Gas phase streamer mechanisms cannot be directly applied to the breakdown phenomena in water since electron impact ionization and photo ionization are insignificant at the breakdown fields. Also, the characteristics of the electrical breakdown, caused by pulsed voltages in tap and distilled water with lower conductivities, have only been partially investigated.

Streamer discharge phenomena in water are known to be influenced by various factors, such as gap geometry, water conductivity, pressure, pulse duration, polarity, and amplitude of the applied voltage [6]-[9]. It is generally assumed that "thermal" processes are associated with microsecond breakdown liquids, and "electron avalanche" processes are

$\dagger$ Corresponding Author: School of Electrical Engineering, Inha University, \#253, Yonghyun-dong, Nam-ku, Incheon, 402-751, Korea . (bhlee@inha.ac.kr)

* School of Electrical Engineering, Inha University, Korea. (chjohy80@naver.com)

Received: January 21, 2010; Accepted: April 27, 2010 closely associated with the underwater discharges over shorter time scales. It is also important to determine whether an electron avalanche can occur in water at the breakdown fields [10]-[12]. The pre-breakdown growth is likely to proceed via electron avalanche in the bubbles that are present in the water [8]-[9]. An issue of fundamental breakdown is whether or not an electron avalanche can occur in the liquid water at the breakdown. Two leading theories of the initial breakdown mechanisms in water are: 1) charge injection at the electrode/water interface, and 2) electron impact ionization in a low-density region of the water [5], [8], [12] The mechanism of the streamer corona initiation and propagation in water is still not well understood, and more quantitative and detailed experimental results have to be determined.

In order to understand clearly the underwater discharge phenomena associated with a streamer initiation, and propagation in the water under impulse voltages, the present work focuses on investigating the streamer corona phenomena, and establishing the important parameters of the underwater electrical discharges under 1.2/50 $\mu$ s impulse voltages, in situations involving point-to-plane electrode systems, together with collecting the visual data and information emitted during the discharge developments. Some measured results and physical properties are also discussed in detail.

\section{Experiments}

\subsection{Experimental Setup}

Fig. 1 shows a schematic diagram of the experimental 
setup, which consists of a Marx generator, a test cell, and electrical and optical signal recording systems. The pointto-plane electrode assembly is completely immersed in the center of the test cell, made of colorless and transparent acrylic board, and the test cell is filled with $8,000 \mathrm{~cm}^{3}$ of water at room temperature. The point electrode is made of a stainless steel $3 \mathrm{~mm}$ diameter needle with a conical end, and the tip radius of curvature is $0.1 \mathrm{~mm}$. The plane electrode is formed by a polished copper disc of a diameter of $100 \mathrm{~mm}$, with a quasi-Rogowski profile, and this is connected to ground via a coaxial shunt of $0.02 \Omega$. The gap distance between the electrodes is $35 \mathrm{~mm}$.

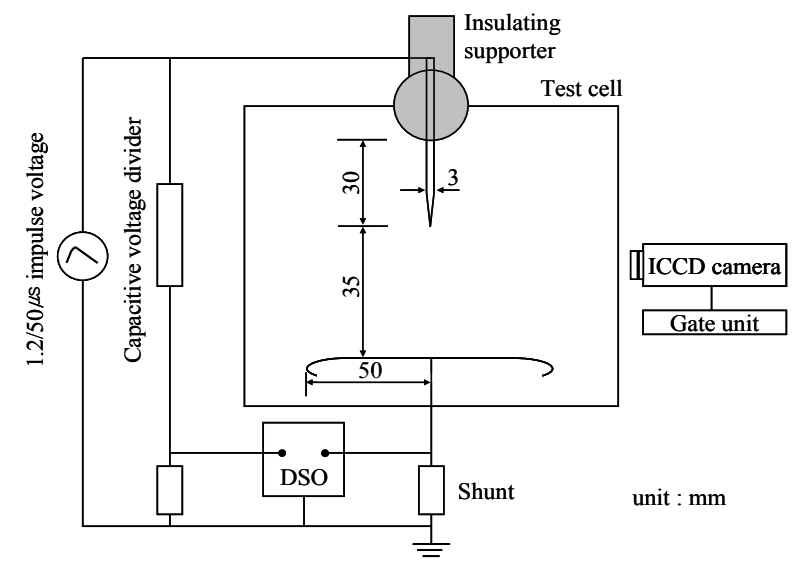

Fig. 1. A schematic diagram of the experimental setup.

In order to investigate the electrical and physical properties of underwater streamer discharges, high-speed devices for measuring the voltage, the current and optical images of the discharges have been used. Positive or negative $1.2 / 50 \mu \mathrm{s}$ impulse voltages are applied to the needle electrode and the test voltages are measured by means of a 10,000:1 damped-capacitive voltage divider. The current is monitored by means of a sensitive current shunt with the step response time of less than $24 \mathrm{~ns}$, and voltage/current waveforms have been recorded using a digital impulse analyzer. The discharge light images are analyzed with long exposure time photographs taken by a digital camera, and short exposure time photographs taken by a high-speed ICCD camera controlled via a gate unit.

\subsection{Methodology}

Distilled water at room temperature with a conductivity of $100 \mu \mathrm{S} / \mathrm{m}$ has been used as the trial solution. The water conductivity, measured via an electric conductivity meter (CM-21, TOA), is controlled by adding $\mathrm{CuSO}_{4} \cdot \mathrm{H}_{2} \mathrm{O}$, which is a good melting material. The light images of the discharge, associated with the pre-breakdown and the breakdown processes, have been taken for different voltages and water conductivities using a digital camera triggered by the applied voltage pulse, and its exposure time set to $10 \mathrm{~ms}$. The pre-breakdown development processes from the streamer corona initiation phase to the stepped leader propagation phase have been analyzed using current oscil- lograms and the light images of the discharges. Various aspects have been investigated, including the electrical breakdown voltage, the streamer propagation velocity, the time-lag to breakdown, and the pre-breakdown energy signatures. The detailed specifications for the main functions of the ICCD camera are listed in Table 1.

The temporal sequence of the signals used to operate the ICCD camera is shown in Fig. 2 where it can be seen that the ICCD camera is triggered after a delay of a fixed time; this is the time required for the ICCD camera to record a light image.

Table 1. Specifications of the ICCD camera used in this work

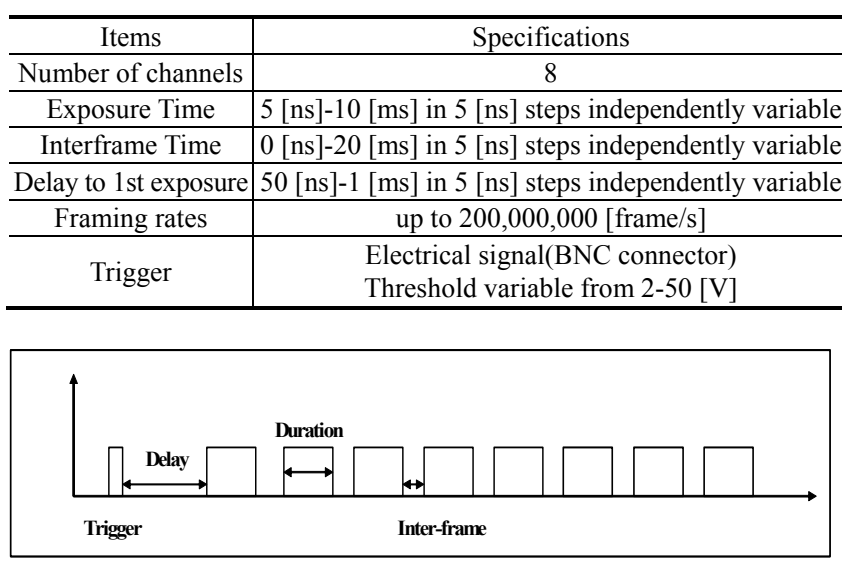

Fig. 2. Time signals to operate the ICCD camera.

\section{Results and Discussion}

\subsection{Pre-breakdown Development Processes}

Electrical breakdown within liquids is generally preceded by events called "streamers". The two possible mechanisms for streamer initiation need to be considered: the first is due to electron avalanche which cause electrons to be injected in the liquid and drift to the cathode, and the other is due to the formation of micro-bubbles which cause gaseous cavities to be formed that give rise to electrical breakdown in the gas bubbles. The electric field around the tip of the needle electrode, or the streamer channel, is highly increased, which causes an intense field emission current that eventually vaporizes the water. Also, the very high electric field causes a cavity due to the electromechanical stress, which is proportional to the square of the electric field strength [9], [13].

When a positive voltage greater than $25 \mathrm{kV}$ is applied, streamers are initiated and propagate towards the cathode. The positive streamer propagates from the anode, and electrons fall into its tip from the water molecules located ahead of it, which become dissociated by the local intense electric field. The negative streamers start from the sharp point on the cathode when the applied voltage is greater than about $20 \mathrm{kV}$. The growth of the negative streamers is 
aided by electrons bombarding the gas-water interface at the tip of the streamers, and this growth of the streamer causes its space charge field to increase exponentially [14].

Fig. 3(a) shows examples of the time-integrated photographs of the streamer discharges in water in the case of the positive or negative impulse voltages being applied to the needle electrode. The filamentary shaped streamers are observable at the tip of the needle electrode, and correspond to sequences of low-current streamer pulses. Streamer coronas develop in the water when a very high electric field is suddenly applied to the water gap causing an electron avalanche to grow directly in the water, leading to the propagation of a water streamer [15]. If the applied voltage is insufficient for bridging the test gap, the streamer coronas can cease, as shown in Fig. 3.

The pre-breakdown current waveforms have been recorded to investigate the characteristics of the streamer corona propagation, and Fig. 3 (b) shows typical examples of the temporal developments of both the voltage and the discharge currents obtained when electrical breakdown does not occur. The streamer corona current pulses appear, after some delay time, when the applied voltage is increased. The streamer current traces are made up of a large number of pulses with amplitude greater than $1 \mathrm{~A}$ which stop after a short time, and do not take place in an electrical breakdown. These current waveforms consist of the conduction current component and form a sequence of short corona current pulses corresponding to the streamer regime, that is, the streamer corona current pulses are superimposed on the conduction current, which is proportional to the applied voltage. The ionic currents make little contribution to breakdown initiation since they play no role in its formation on the short time scales [8].

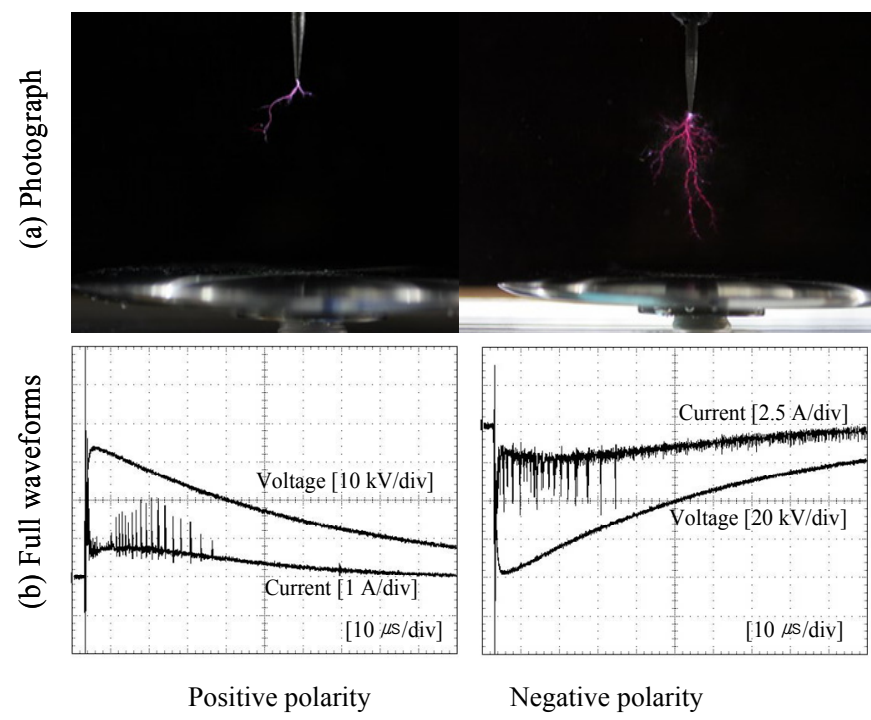

Fig. 3. Time-integrated photographs of the streamer discharges and the corresponding voltage/current oscillograms for water conductivity of $100 \mu \mathrm{S} / \mathrm{m}$.

The initial oscillations of the current waveforms are attributed to a capacitive effect due to the fast rise of the ap- plied voltage. Below the breakdown voltages, the stepped leader process ceases after several steps. The time intervals between streamer corona steps decrease with voltage, and are distributed in the range 1-5 $\mu \mathrm{s}$. The numbers of streamer current pulses for the negative polarity are much greater than those in the positive polarity. Consequently, the pre-breakdown development is initiated by the streamer corona and the streamer to leader transition takes place after a dead time and this discharge process is repeated. Two physical processes, namely the ionic drift and the channel expansions occur during the pause time between the current pulses [16], [17].

A number of streamer corona branches appear as the applied voltage increases. Above the breakdown voltage level, the fastest streamers are able to bridge the test gap and to cause arc formation. Fig. 4 displays typical voltage/current waveforms recorded when breakdown has occurred.

The sequence of corona current pulses corresponds to the streamers propagating towards the plane electrode. In the presence of both the positive and negative polarities, it is clear that electrical breakdowns occur due to the stepped leader propagation. The pre-breakdown processes are successively sustained by a stepped leader regime to the breakdown. The streamer current pulses take place intermittently, and the time interval between the corona current pulses indicates an extensive stepped leader regime. The amplitudes of the streamer corona current pulses are very irregular, and the time intervals between streamer current pulses are seen to be distributed in the range 1-2 $\mu$ s.

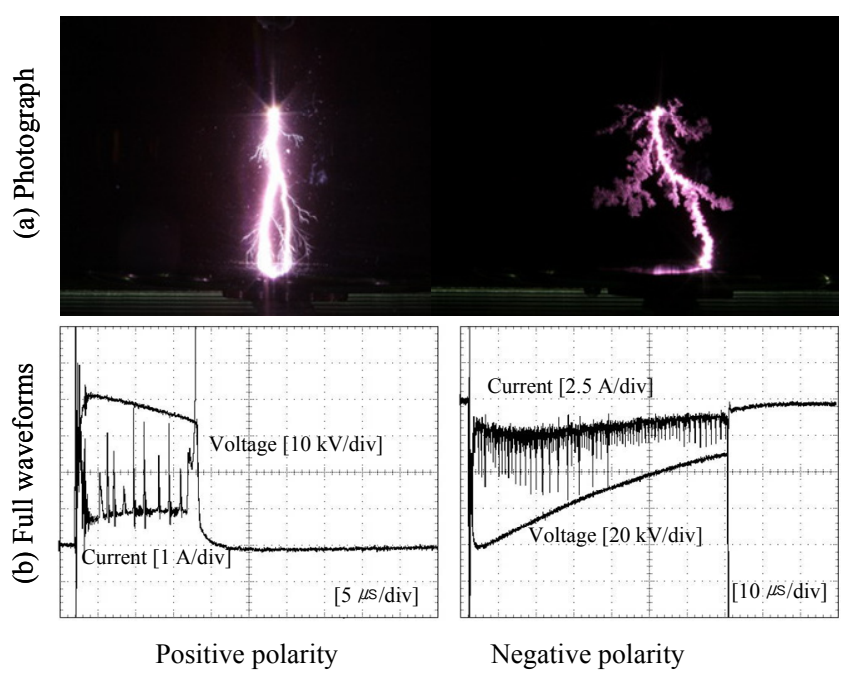

Fig. 4. Time-integrated photographs of streamer discharges and the corresponding voltage/current oscillograms when the water conductivity is $100 \mu \mathrm{S} / \mathrm{m}$.

The overall shapes of the positive pre-breakdown current waveforms are similar to those of the negative streamer current pulses, which are much greater than the positive currents. In the case of the negative polarity, the amplitude of the streamer corona current is increased at the beginning part of the pre-breakdown, then decreases after some extension, followed by an increase just before the breakdown. 
The time intervals between the streamer corona pulses in the negative polarity are similar to those in the positive polarity, it appears that the pre-breakdown development mechanisms for both the positive and negative polarities are basically the same.

The underwater breakdown is principally an electrical discharge process caused by the avalanche effect of the air in the bubbles. When a very high electric field is rapidly applied at the tip of the needle electrode in the water, the thermal process leads to the creation of air bubbles in which electrical discharges develop. When the streamer corona is initiated in the water, air bubbles are generated, which first expand and later collapse. It is found that the lower the water conductivity, the lower the streamer inception voltage. The underwater breakdown mechanism associated with the ionization of the gas in the bubbles has been previously reported by some researchers (see for example [3], [18], [19]).

In order to investigate the temporal development of the streamer corona discharges in water, a fast gated ICCD camera has been employed, and the temporal development of the optical emission images, after initiation of the streamer corona, has been observed and recorded. The camera is triggered by the signal, which detects the charging voltage of the Marx generator and after a delay to the first exposure for a fixed time according to the time setting conditions, eight frames of the temporal development of the streamer corona discharge can be consecutively recorded with the ICCD camera. Fig. 5 shows examples of the timeintegrated photographs of some streamer discharge light images, recorded by the ICCD camera.

Fig. 5(a) illustrates the growth process of a positive streamer in water when a voltage of $37 \mathrm{kV}$ is applied 3.2 $\mu \mathrm{s}$ after the first frame. The exposure time of the frame is $0.2 \mu \mathrm{s}$ and the inter-frame time is zero. The shutter time of the ICCD camera has been set to $1 \mathrm{~ns}$, and the optical setting of the camera, including the intensification gain, is kept the same for each photograph. Streamers in liquids are generally classified as fast and "filamentary" for streamers emanating from the positive electrode, or slow and "bushlike" for streamers emanating from the negative electrode [14]. Many streamer branches have been observed, and the streamer propagation length increases linearly with time. In the case that the positive impulse voltage is applied to the needle electrode, after a time of $3.2 \mu \mathrm{s}$ the streamer coronas are initiated at the tip of the needle electrode, and they begin to propagate toward the plane electrode. It is obvious that the light image of the streamer coronas is brighter and the streamer channel is thicker near the tip of the needle electrode.

Fig. 5(b) illustrates the growth process of a negative streamer in water; the first frame is taken at a voltage of 85 $\mathrm{kV}$, after a delay of $3 \mu \mathrm{s}$ from the origin. The exposure time of the frame and the inter-frame time are $2 \mu \mathrm{sand} 7 \mu \mathrm{s}$, respectively. The last frame is taken with a long inter-frame time to record the final jump image bridging the test gap. Each of the streamer coronas consists of many branch channels spreading towards the plane electrode, and all the branch channels develop radially. The number of negative streamer branches is much larger than that of the positive streamer branches. The light images emitted by the streamer discharge developments are generally similar from frame to frame. The traces of the consecutive streamers from the needle electrode or the leader channel tip are not of equal length but, the leader streamer does move persistently into the test gap. In addition, the streamer corona branches in water of higher conductivities are much thicker and brighter than those in lower conductivities at the same applied voltage levels.
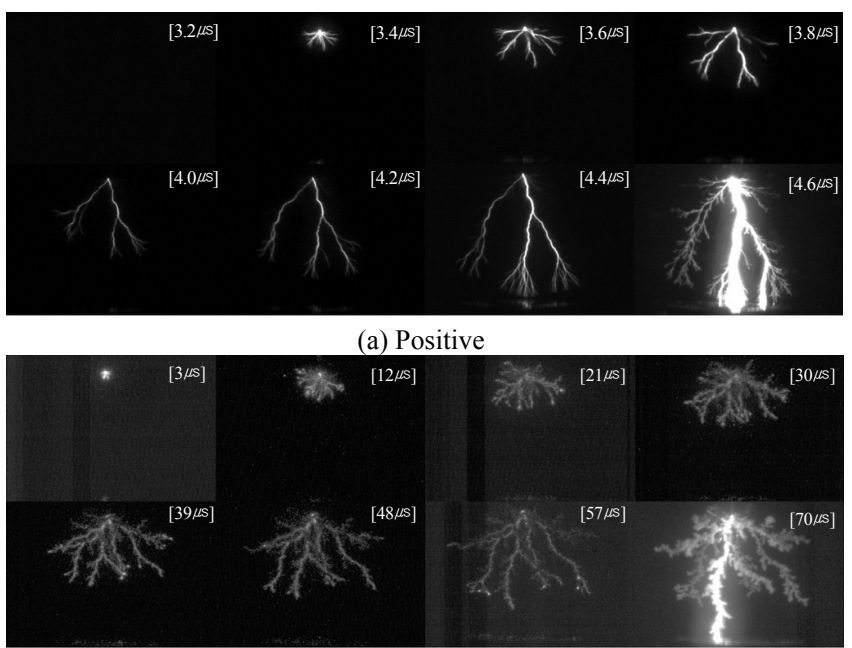

(b) Negative

Fig. 5. Typical examples of the time-integrated photographs of streamer discharge light images observed by the ICCD camera when the water conductivity is $100 \mu \mathrm{S} / \mathrm{m}$.

The propagation length of the streamer corona discharge around the tip of the needle electrode, in radial directions, is not uniform, as shown in Fig. 5. The streamer corona expands intensively in the axial direction of the needle electrode, in comparison with the lateral direction, because of the conical tip end of the needle electrode. The propagation length of the visual streamer corona increases with increasing applied voltage until electrical breakdown occurs, as reported in the literature [6]. The streamer propagation length is directly obtained from the time-resolved photographs taken by the ICCD camera, and Fig. 6 shows the variation of the maximum streamer propagation length for different water conductivities as a function of peak voltage.

The propagation length of the positive streamer corona is very erratic, irrespective of water conductivity. Although the data in the negative polarity are dispersed, the propagation length of the streamer coronas is linearly proportional to the peak voltage. During the propagation of the streamer, numerous micro-bubbles are produced, not only in the vicinity of the stem of the leader but also at the tip of the streamer. The size of bubble must be sufficient for growth of the streamer corona and the conversion into a fast breakdown streamer, according to the theory of discharge in liquids in microseconds [5]. 


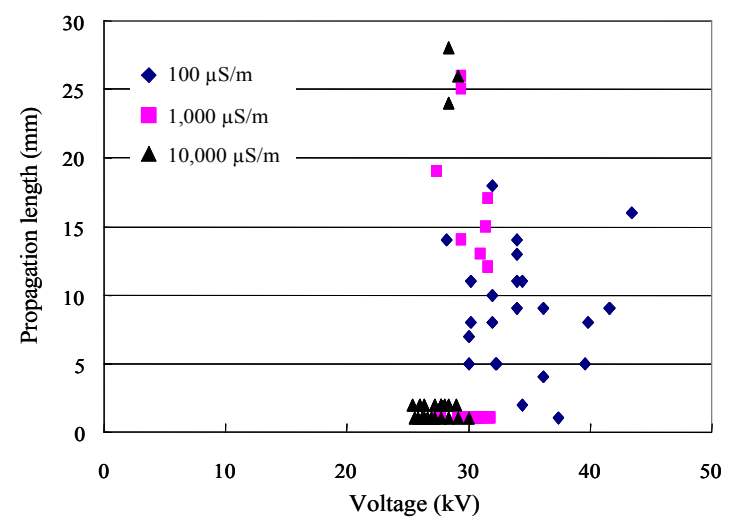

(a) Positive

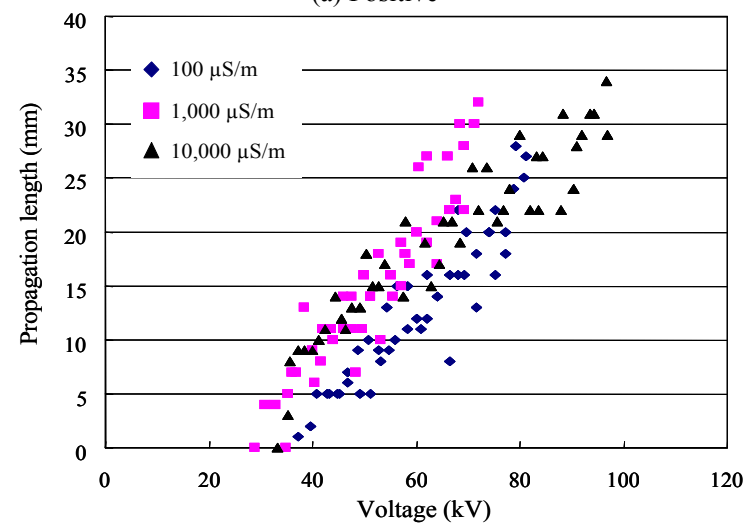

(b) Negative

Fig. 6. Propagation length of the streamer corona as a function of applied voltage

The difference between streamer propagation lengths in consecutive photographs corresponds to streamer extension during the inter-frame time. From these measurements, the average streamer propagation velocity can be calculated, and Fig. 7 illustrates the average streamer propagation velocity as a function of water conductivity.

Positive streamers with an average propagation velocity of about $2.9 \mathrm{~km} / \mathrm{s}$ have been observed, but the average propagation velocity for the negative streamers is found to be approximately $0.5 \mathrm{~km} / \mathrm{s}$. The average propagation velocities for both the positive and negative streamers are found to be independent of the water conductivity. The average propagation velocity during steps for positive streamers is approximately 60 times faster than that for negative streamers, but it is much less than the drift velocity for electrons in water vapor at room temperature [20].

Katsuk, et al. [9] have reported that the propagation velocities of positive streamer discharges between wire and plane electrodes in tap and distilled water have almost the same values of $32 \mathrm{~mm} / \mu \mathrm{s}$, despite the different appearances of the discharges. In addition, the propagation velocity stays almost constant during the time that the voltage is applied, despite the fact that the distance between the streamer head and the plane electrode reduces with time. The average streamer propagation velocity is constant during the time that the voltage is applied and has almost identical values for different voltages. This effect is explained by considering the energy flow into the streamer head and its effect on the thermal expansion of the head [9].

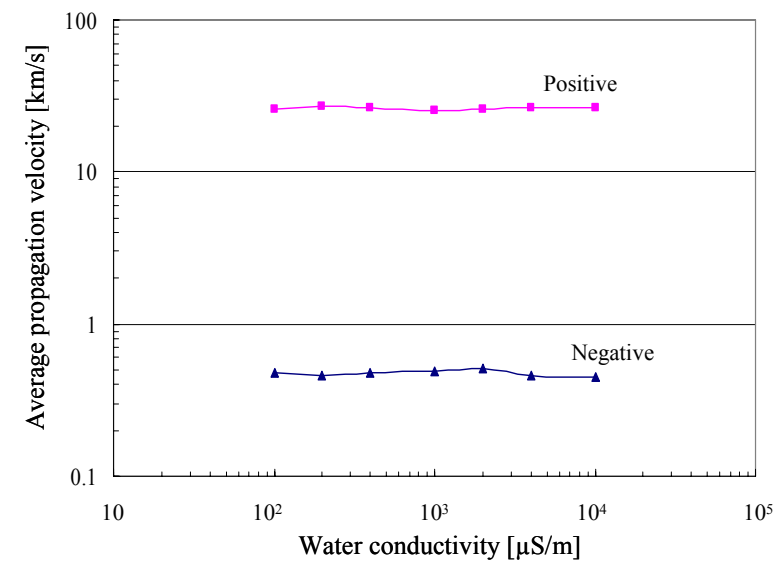

Fig. 7. Average propagation velocities of the streamers as a function of water conductivity.

The slow streamer can indicate a streamer filled with microbubbles of multiple sizes, that is, new phases of water are formed. The mechanism of the bubble formation is gaseous cavitations, which may develop due to Coulomb forces. The first microbubbles may originate from gas adsorbed and absorbed on the electrodes, gas dissolved in water, and gas formed by water dissociation. This new phase is charged, and the carriers can originate from the microbubbles after being ionized and the internal gas being discharged [21].

\subsection{Breakdown Characteristics}

The dependence of the breakdown voltage on the conductivity of the water has been investigated in the current research. The underwater breakdown, caused by the impulse voltage, is not stable because of the randomness of the streamer corona propagation. The electrical breakdown voltage is determined by the up and down method, with using a voltage difference of $2 \mathrm{kV}$; Fig. 8 shows the impulse breakdown voltages against water conductivity for both positive and negative polarities. The negative breakdown voltages are significantly higher than the positive breakdown voltages and the positive breakdown voltages appear to be inversely proportional to the water conductivity. The breakdown voltage - water conductivity curve for the negative polarity, shows a U-shape, and the minimum breakdown voltage appears when the water conductivity is around $2,000 \mu \mathrm{S} / \mathrm{m}$.

It is demonstrated that the polarity effects on the electrical breakdown of liquids basically arise from the large mobility difference between electrons and ions. The higher electron mobility leads to greater charge smearing and diffusion which influences the electric field distributions. Non-linear coupling between the number density, electric field and charge production rates then collectively affects 
the formation and propagation of ionized channels, and influences the light emission characteristics [22].

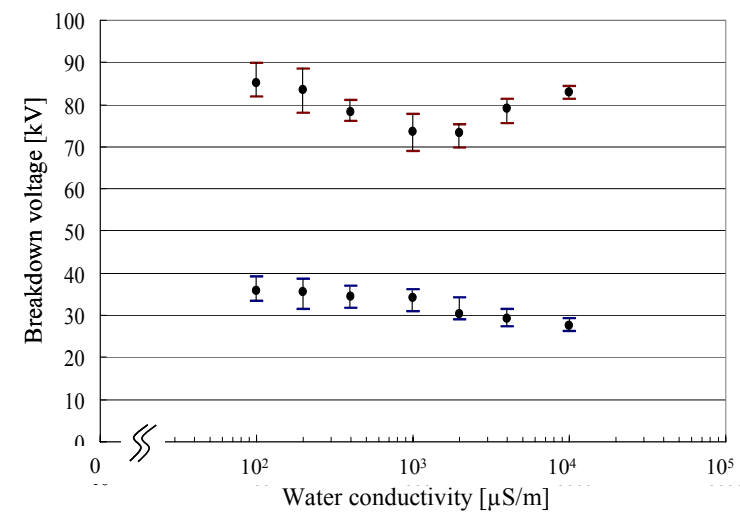

Fig. 8. Impulse breakdown voltages versus water conductivity.

Fig. 9 shows the breakdown voltage - time $(V-t)$ characteristics for the test gap, where the breakdown voltage is taken as the peak value of the applied voltage, and the time is the time-lag to the breakdown. This time-lag to breakdown is strongly dependent on the polarity of the applied voltage, and is insensitive to water conductivity. The timelags to breakdown under positive polarities are much shorter than for the negative breakdown ones. The time to breakdown in the positive and negative polarity cases ranged from about $2 \mu \mathrm{s}$ to $30 \mu \mathrm{s}$, and from $55 \mu \mathrm{s}$ to 120 $\mu \mathrm{s}$, respectively. The $V-t$ curves for the lower water conductivities lay in the upper part in a longer time range.

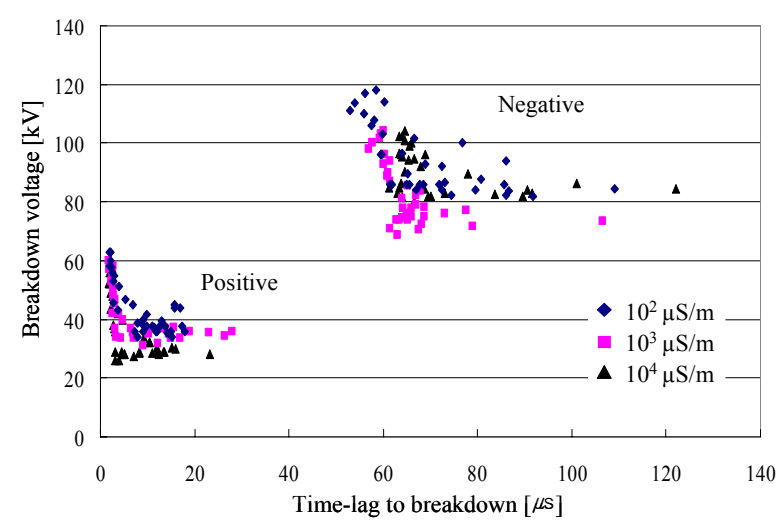

Fig. 9. Breakdown voltage-time characteristic curves as parameters of the water conductivity and the polarity of applied voltage.

Also, the scatter of the breakdown voltages in the negative polarity cases are distinguished, and may arise from many factors, including random variations in the mean speed between leaders, the streamer corona progression and the avalanche effects in the microbubbles during the pre-breakdown processes.

The system for measuring and analyzing impulse voltage and current is suitable for estimating the energy injected from the source to the test gap, prior to the breakdown. Typical voltage, current, power, and energy waveforms obtained from the impulse measuring and analyzing program for the water conductivity of $10,000 \mu \mathrm{S} / \mathrm{m}$ in the negative polarity are shown in Fig. 10, where the delay to the electrical breakdown suggests that there is a critical energy for the streamer bridging the test gap.

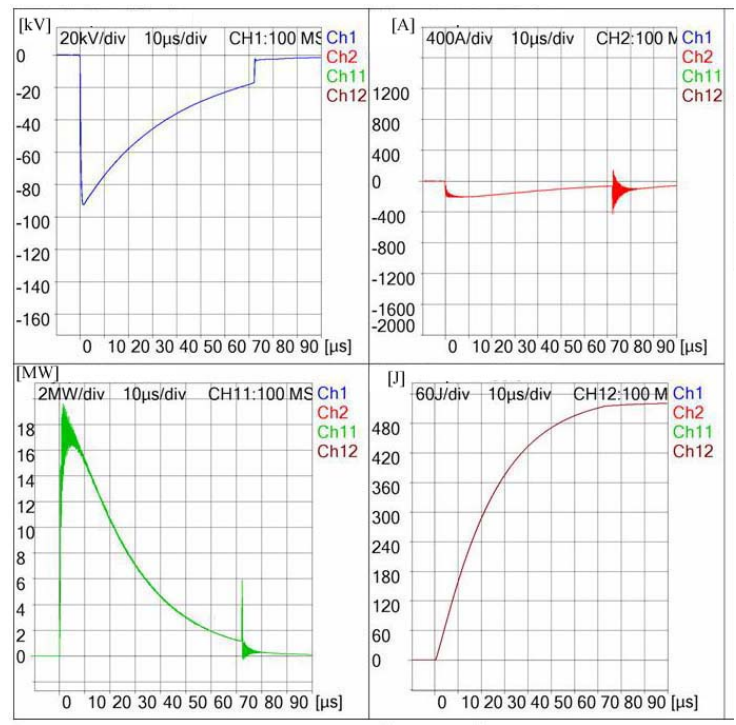

Fig. 10. Typical voltage, current, power and energy waveforms obtained from the impulse measuring and analyzing program for the water conductivity of $10,000 \mu \mathrm{S} / \mathrm{m}$ in the negative polarity.

Fig. 11 illustrates the relation between the prebreakdown energy and the water conductivity. The prebreakdown energy for both the positive and negative polarities was nearly proportional to the water conductivity. Unfortunately, there is no measurable energy from the prebreakdown current pulses, excluding the ionic current. The pre-breakdown energy strongly depends on the ionic current flow, which is approximately linearly proportional to the water conductivity. Because the same conduction current flows through the test gap before breakdown in higher water conductivity, the pre-breakdown energy is much more than that in lower water conductivities. In the case of the same water conductivity, the negative breakdown voltages are much higher than the positive breakdown voltages.

Also, the negative time-lags to breakdown are much longer than the positive time-lags to breakdown, as shown in Fig. 9, so it is considered that the negative prebreakdown energy is significantly increased by the accumulation effect of the longer time-lag to breakdown and the higher breakdown voltage. It is known from the insensitivity of the time-lag to the breakdown on the water conductivity that the bulk and local heating by ionic prebreakdown currents does not contribute much to the initiation of the breakdown process [10]. 


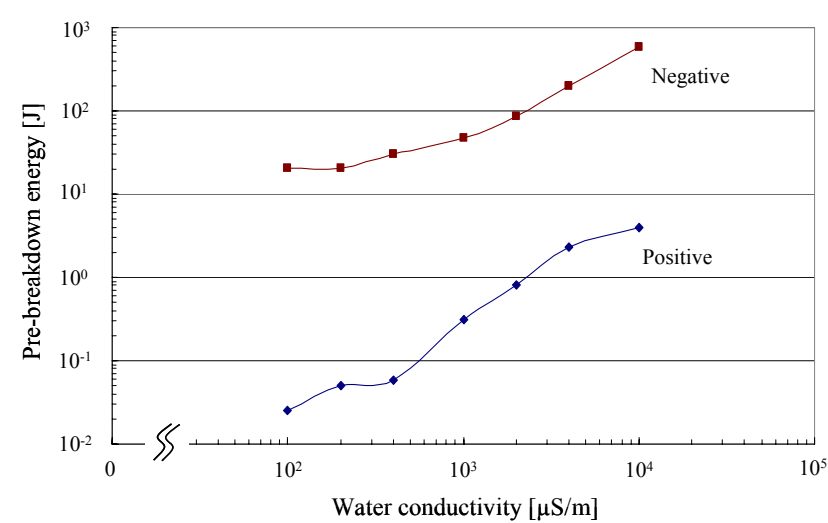

Fig. 11. Pre-breakdown energy as a function of the water conductivity.

Some energy deposited into the test gap during the prebreakdown streamer corona propagation is required for the formation of microbubbles at the tip of the needle electrode. The size of the microbubbles must be sufficient for growth of the streamer, according to the theory of discharges in liquids in the microsecond timescales [9], [23].

The sequence of current pulses corresponds to streamer corona propagating towards the plane electrode. During the streamer corona period of discharge developments, gaseous bubbles are formed at the tip of the needle electrode [23]. The pre-breakdown streamer corona is followed by the formation of stepped leaders propagating towards the plane electrode [24]. The stepped leaders propagate as two modes, namely, (a) rapid development of the ionization zone at the tip of the leader channel, and (b) a pause at the end of which the previous ionization zone is transformed into a new section of the leader channel, accompanied by the formation of a new ionization zone [12], [21], [23]-[24].

\section{Conclusions}

The temporal behaviours of streamer corona initiation and propagation has been observed and investigated to characterize underwater pre-breakdown and the breakdown processes in highly inhomogeneous electric fields under impulse voltages. The results presented show that the succession of streamer coronas during pre-breakdown produces a stepped propagation of leaders. Above the breakdown voltage level, the stepped leaders propagate up to the plane electrode after some steps, and breakdown takes place. The pre-breakdown currents consist of the conduction current and streamer corona current pulses. It is found that the avalanche effect of the gas in the bubbles plays a dominant role in initiating and developing the streamer coronas and the stepped leaders. The underwater breakdown voltages strongly depend on the polarity of the applied voltage. The average streamer propagation velocity for both the positive and negative polarities does not appear to depend on the water conductivity. The breakdown voltages and time-lags to breakdown in the negative polarity are significantly higher and longer compared with the data in the positive polarity situations.

\section{Acknowledgements}

This work was supported by the Ministry of Knowledge Economy under Grant R-2007-1-014.

\section{References}

[1] K. Arii, I. Kitani and M. Kawamura, "Avalanche Breakdown in Liquid n-Hexane," J. Phys. D: Appl. Phys., Vol. 12, pp. 787-796, 1979.

[2] R. P. Joshi, J. Qian and K. H. Schoenbach, "Electrical Network-based time-dependent model of electrical breakdown in Water," J. Appl. Phys., Vol. 92, No. 10, pp. 6245-6251, 2002.

[3] X. Lu, Y. Pan, K. Liu, M. Liu and H. Zhang, "Spark model of pulsed discharge in water," J. Appl. Phys., Vol. 91, No. 1, pp. 24-31, 2002.

[4] H. Akiyama, "Streamer discharges in liquids and their applications," IEEE Trans. DEI, Vol. 7, No. 5, pp. 656-653, 2000.

[5] A. T. Sugiarto, M. Sato and J. D. Skalay, "Transient regime of pulsed breakdown in low-conductive water solutions," J. Phys. D, Vol. 34, pp. 3400-3406, 2001.

[6] P. Bruggeman, C. Leys and J. Vierendeels, "Experimental investigations of dc electrical breakdown of long vapour bubbles in capillaries," J. Phys. D: Appl. Phys., Vol. 40, pp. 1937-1943, 2007.

[7] Y. H. Sun, Y. X. Zhou, M. J. Jin, Q. Liu and P. Yan, "New prototype of underwater sound source based on the pulsed corona discharge," J. Electrostatic, Vol. 63, pp. 969-975, 2005.

[8] H. M. Jones and E. E. Kunhardt, "The influence of pressure and conductivity on the pulsed breakdown of water," IEEE Trans. DEI, Vol. 1, No. 6, pp. 10161025, 1994.

[9] S. Katsuki, J. Akiyama, A. Abou-Ghazala and K. H. Schoenbach, "Parallel Streamer Discharges Between Wire and Plane Electrodes in Water," IEEE Trans. DEI, Vol. 9, No. 4, pp. 498-506, 2002.

[10] K. Yoshino, "Electrical Conduction and Dielectric Breakdown in Liquid Dielectrics," IEEE Trans. on Electrical Insulation, Vol. 21, pp. 847-853, 1986.

[11] P. K. Watson, "Electrostatic and Hydrodynamic Effects in the Electrical Breakdown of Liquid Dielectrics," IEEE Transaction on Electrical Insulation, Vol. 20, pp. 395-399, 1985.

[12] D. A. Wetz, K. P. Truman, J. J. Mankowski and M. Kristiansen, "The Impact of Surface Conditioning and Area on the Pulsed Breakdown Strength of Water," IEEE Trans., on Plasma Science, Vol. 33, No. 4, pp. 1161-1169, 2005.

[13] T. J. Lewis, "A New Model for the Primary process of Electrical Breakdown in Liquids," IEEE Trans. DEI, Vol. 5, No. 3, pp. 306-315, 1998. 
[14] M. Abdel-Salam and A. El-Morshedy "High-Voltage Engineering Theory and Practice," Marcel Dekker, pp. 123-234, 2000.

[15] G. Touya, T. Reess, L. Pecastaing, A. Gibert and P. Momens, "Development of subsonic electrical discharges in water and measurements of the associated pressure waves," J. Phys. D, Vol. 39, pp. 5236-5244, 2006.

[16] B. H. Lee, Y. H. Baek, H. S. Choi and S. K. Oh, "Impulse breakdown characteristics of the plane-to-plane electrode system with a needle-shaped protrusion in SF $_{6}$," Current Applied Physics, Vol. 7, pp. 289-295, 2007.

[17] T. Kawamura and B. H. Lee, "Transient Impulse breakdowns of $\mathrm{SF}_{6}$ Gas in Inhomogeneous Electric Fields," Jpn. J. Appl. Phys. Vol. 38, part 1, No. 8, pp. 4898-4904, 1999.

[18] J. Nieto-Salazar, O. Lesaint and A. Denat, “Transient current and light emission associated to the propagation of pre-breakdown phenomena in water," Proc. 2003 Annual Report Conference on Electrical Insulation and Dielectric Phenomena, pp. 542-545, 2003.

[19] I. M. Gavrilov, V. R. Kukhta, V.V. Lopatin and P.G. Petrov, "Dynamic of Prebreakdown Phenomena in a Uniform Field in Water," IEEE Trans. DEI, Vol. 1, No. 3, pp. 496-502, 1994.

[20] A. Gilardini, Low Energy Electron Collisions in Gases: Swarm and Plasma Methods Applied to Their Study, John Wiley \& Sons, pp. 409-413, 1972.

[21] V. R. Kukhta and V. V. Lopatin, "Inception and Development Breakdown Cavities in Water," Proc. 13th International Conference on Dielectric Liquids, Nara, Japan, pp. 273-276, 1999.

[22] J. Qian, R. P. Joshi, Z. Schamiloglu, J. Gaudet, J. R. Woodworth and J. Lehr, "Analysis of polarity effects in the electrical breakdown of liquids," J. Phys. D, Vol. 39, pp. 359-369, 2006.

[23] H. M. Jones, E. E. Kunhardt, "Pulsed dielectric breakdown of pressurized water and salt solutions," $J$. Appl. Phys., Vol. 77, No. 2, pp. 795-805, 1995.

[24] B. H. Lee, "Breakdown Behavior of $\mathrm{SF}_{6}$ Gas in Nonuniform Electric Field under Transient Impulse Voltages," J. Korean Physical Society., Vol. 34, No. 3, pp. 248$253,1999$.

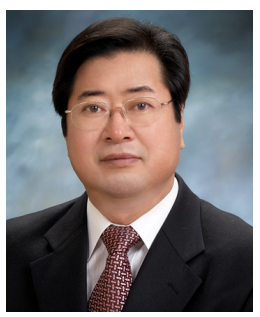

Bok-Hee Lee received his Ph.D. degree in Electrical Engineering from Inha University in 1987. He has been with the school of Electrical Engineering at Inha University, Incheon, Korea, as a Assistant Professor in 1990, where he became a Professor in 1999. From 1988 to 1989 , he was a post-doctoral research fellow at the Institute of Industrial Science, University of Tokyo. From Apr. 1999 to Feb. 2000, he was a Visiting Professor at the University of Cincinnati. Since Oct. 2002, he has been a Director in the Research Center for High-voltage and Power Technology, Inha University. His research interests are in the area of lightning, lightning protection, grounding systems, surge protection, high voltage engineering and electromagnetic compatibility.

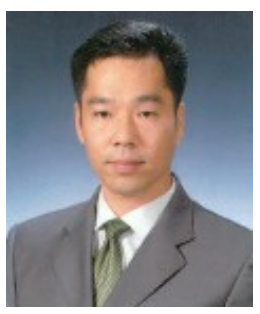

Dong-seong Kim was born in Korea on March 1, 1970. He received his M.S. degree from the Department of Electrical Engineering at Inha University in 2006. He is currently working toward a Ph.D. degree in Electrical Engineering at Inha University. He has been working as a manager at Korea Electrical Safety Corporation. His research interests are in the area of electrical safety, lightning, grounding, electrotechnical regulation and high voltage engineering.

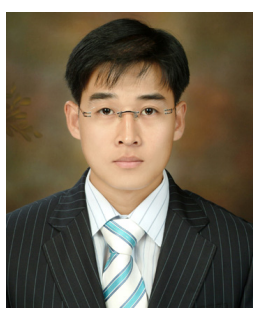

Jong-Hyuk Choi was born in Incheon, Korea, in 1980. He received his B.S. degree in Electronic Engineering from Inha University in 2006. He received his M.S. degree in Electrical Engineering from Inha University in 2008. He is currently working toward his Ph.D. degree at the School of Electrical Engineering, Inha University, Incheon, Korea. His research interests include high voltage engineering, grounding, and discharge phenomena. 